\title{
High Molecular Weight Poly(vinyl alcohol) through Photo-Emulsion Polymerizations of Vinyl Acetate
}

\author{
Tohei Yamamoto, Shigetoshi Seki, Ryohei Fukae,* \\ Osamu SANGEN, and Mikiharu KaMACHI** \\ Himeji Institute of Technology, Shosha, Himeji 671-22, Japan \\ *Himeji Junior College, Shinzaikehonmachi, Himeji 670, Japan \\ **Faculty of Science, Osaka University, Machikaneyama, \\ Toyonaka 560, Japan
}

(Received October 6, 1989)

\begin{abstract}
A mixture of vinyl acetate, water and polyoxyethylene nonyl phenyl ether sodium sulfate (LWZ; Kao Co.) was irradiated with a high pressure mercury lamp in the absence of initiator at $0^{\circ} \mathrm{C}$ and high molecular weight poly(vinyl acetate) (PVAc) which gave poly(vinyl alcohol) (PVA) of $P_{n}$ (average degree of polymerization) $=12800$ after hydrolysis was obtained in good yield. Initiation by a radical from LWZ decomposed by photoirradiation was deduced. The charateristics of the PVA were similar to those of usual PVA.
\end{abstract}

KEY WORDS High Molecular Weight / Poly(vinyl alcohol) / Photo-

Emulsion Polymerization / Vinyl Acetate / Non-Initiator /

Poly(vinyl alcohol) (PVA) fiber spun from high molecular weight PVA gel is expected to have high modulus and high strength. ${ }^{1}$ However, it is very difficult to obtain PVA of high molecular weight, because chain transfer reactions control the molecular weight of polymers in radical polymerization of vinyl acetate (VAc), which are precursors for PVA. Moreover, degradation occurs by hydrolysis of branched poly(vinyl acetate) (PVAc) formed by chain transfer reaction. ${ }^{2}$

In order to obtain a high molecular weight polymer, emulsion polymerizations of VAc were examined at low temperature. In the course of study it was found that some of them gave PVA of which $P_{n}$ (average degree of polymerization) was over $1 \times 10^{4}$ after hydrolysis of PVAc. A part of the results has been reported $^{3)}$ and further detailed results are shown in the present paper.

\section{EXPERIMENTAL}

\section{Materials}

VAc was washed with aqueous solution of $\mathrm{NaHSO}_{3}$ and water, dried over anhydrous $\mathrm{CaCl}_{2}$ and distilled under reduced pressure of nitrogen. Distilled water was used as an medium. Polyoxyethylene nonyl phenyl ether sodium sulfate (LWZ; Kao Co.) and sodium dodecyl sulfate (SDS) were used as emulsifiers without further purification. Potassium persulfate (KPS) and 2,2-azobis(2-amidinopropane)hydrochloride (AAPH) were purified by recrystallization and used as initiators.

\section{Polymerizations}

In a $30 \mathrm{ml}$ Pyrex Kjeldahl flask were placed $5 \mathrm{ml}$ of VAc, a fixed amount of emulsifier, $10 \mathrm{ml}$ of water and a teflon stirring bar. In some cases small amounts of initiator were added. The mixture was degassed and the flask was sealed under vacuum. The flask was placed in a water-ethylene glycol bath $\left(-5 \sim 5^{\circ} \mathrm{C}\right)$, and the 
mixture was stirred and irradiated with a high pressure mercury lamp $(300,313$, and $365 \mathrm{~nm})$ for $10 \mathrm{~h}$. Then the mixture was poured into $150 \mathrm{ml}$ of water, and with stirring $\mathrm{Na}_{2} \mathrm{SO}_{4}$ was added gradually until the emulsion broke and polymer precipitated. After filtration the PVAc was washed with water and dried under vacuum at $60^{\circ} \mathrm{C}$.

\section{Saponification of PVAc}

To a solution of $2 \mathrm{~g}$ of PVAc in $100 \mathrm{ml}$ of methanol was added $2.5 \mathrm{ml}$ of $40 \% \mathrm{NaOH}$ aqueous solution, and the mixture was stirred for $5 \mathrm{~h}$ at room temperature to yield PVA by saponification. The PVA was filtered, washed well with methanol and dried under vacuum at $60^{\circ} \mathrm{C}$.

\section{Acetylation of PVA}

In a three necked flask were placed $1 \mathrm{~g}$ of PVA, $2 \mathrm{ml}(25 \mathrm{mmol})$ of pyridine, $20 \mathrm{ml}$ $(0.2 \mathrm{~mol})$ of acetic anhydride, and $20 \mathrm{ml}$ $(0.35 \mathrm{~mol})$ of acetic acid. The mixture was stirred at $100^{\circ} \mathrm{C}$ for $24 \mathrm{~h}$ under nitrogen to afford PVAc. Then the mixture was poured into cool water and PVAc precipitated. The polymer was filtered, purified to repeat reprecipitation with methanol and water and dried under vacuum at $60^{\circ} \mathrm{C}$.

\section{Determination of $P_{n}$}

$P_{n}$ of PVAc was determined from the intrinsic viscosity $([\eta])$ in benzene at $30^{\circ} \mathrm{C}$ according to eq $1 . P_{n}$ of PVA was determined to be equal to that of acetylated PVA.

$$
\log P_{n}=3.31+1.61 \log [\eta]
$$

\section{${ }^{1} H$-NMR Spectroscopy}

The ${ }^{1} \mathrm{H}$ NMR spectra of PVA were measured by using a JEOL JNM GX-500. The triad tacticity, 1,2-glycol sequences and degree of saponification were determined from the spectra.

\section{RESULTS AND DISCUSSION}

\section{Polymerization by Using KPS}

Emulsion polymerizations of VAc were carried out at $5^{\circ} \mathrm{C}$ using KPS as an initiator by photo irradiation with a high mercury pressure lamp in a bath. As shown in Table I (1-4), polymers of $P_{\mathrm{n}}=7900-15600$ were obtained in good yields. By saponification of the polymers, PVAs of $P_{n}=4900-6300$ were obtained.

\section{Polymerization without Initiator}

As shown in Table I(1-4), PVAc of smaller $P_{n}$ was obtained at higher concentration of LWZ. Since it was considered that LWZ worked as an photo-initiator, emulsion polymerizations of VAc in the absence of initiator were examined at $5^{\circ} \mathrm{C}$. As shown in Table I(5), PVAc was obtained in good yields, but $P_{n}$ was not large. Then polymerization was examined at $5^{\circ} \mathrm{C}$ by irradiation with a lamp outside of the bath to decrease the illumination intensity. The yield decreased but $P_{n}$ increased (Table I(6)).

In order to get PVAc of higher $P_{n}$, polymerization temperature should be lowered to decrease chain transfer reaction. Emulsion polymerizations by irradiation from outside of the bath were examined at $0^{\circ} \mathrm{C}$ without initiator. The results are listed in Table I $(7-12)$, and showed that the yield increased with increasing $\mathrm{LWZ}$ amount up to $0.5 \mathrm{ml}$ and reached to about $80 \%$ with more than $0.5 \mathrm{ml}$ of LWZ. $P_{n}$ of PVAc was 26300 at $0.1 \mathrm{ml}$ of LWZ and decreased gradually with increasing LWZ amount. $P_{n}$ of PVA was 12800 below the amount of $L W Z$ of $0.1 \mathrm{ml}$ and decreased gradually with increasing $\mathrm{LWZ}$ amount.

\section{Dependence on Temperature}

In order to decrease chain transfer reaction and to increase $P_{n}$ of PVA, emulsion polymerization was carried out at $-5^{\circ} \mathrm{C}$. In this case, $2 \mathrm{~g}$ of $\mathrm{NaCl}$ was added in the emulsion to prevent freezing. By irradiation for $10 \mathrm{~h}$, yield was $30 \%$ and $P_{n}$ of PVAc and PVA were 
High Molecular Weight PVA through Photo-Emulsion Polymerizations of VAc

Table I. Results of photo-emulsion polymerizations of VAc

\begin{tabular}{|c|c|c|c|c|c|c|c|c|c|c|}
\hline \multirow{2}{*}{ Run } & \multicolumn{2}{|c|}{ Initiator } & \multicolumn{2}{|c|}{ Emulsifier } & \multirow{2}{*}{$\frac{\mathrm{NaCl}}{\mathrm{g}}$} & \multirow{2}{*}{$\frac{\text { Temp }}{{ }^{\circ} \mathrm{C}}$} & \multirow{2}{*}{$\frac{\text { Time }}{\mathrm{h}}$} & \multirow{2}{*}{$\frac{\text { Yield }}{\%}$} & \multicolumn{2}{|c|}{$P_{n} \times 10^{-4}$} \\
\hline & Type & $\mathrm{moll}^{-1}$ & Type & $\mathrm{ml}$ & & & & & PVAc & PVA \\
\hline $1^{\mathrm{a}}$ & KPS & $1.8 \times 10^{-3}$ & LWZ & 0.6 & 2 & 5 & 4 & 94.0 & 1.56 & 0.63 \\
\hline $2^{\mathrm{a}}$ & KPS & $1.8 \times 10^{-3}$ & LWZ & 1.5 & 2 & 5 & 4 & 92.0 & 1.05 & 0.54 \\
\hline $3^{a}$ & KPS & $1.8 \times 10^{-3}$ & LWZ & 2.0 & 2 & 5 & 4 & 80.0 & 0.81 & 0.49 \\
\hline $4^{a}$ & KPS & $1.8 \times 10^{-3}$ & LWZ & 2.5 & 2 & 5 & 4 & 97.0 & 0.79 & 0.50 \\
\hline $5^{\mathrm{a}}$ & & - & LWZ & 1.5 & 0 & 5 & 4 & 96.0 & 0.91 & 0.58 \\
\hline 6 & & - & LWZ & 1.5 & 0 & 5 & 4 & 73.0 & 1.30 & 0.88 \\
\hline 7 & & - & LWZ & 0.05 & 0 & 0 & 10 & 34.5 & 2.10 & 1.28 \\
\hline 8 & & - & LWZ & 0.1 & 0 & 0 & 10 & 69.0 & 2.63 & 1.28 \\
\hline 9 & & - & LWZ & 0.3 & 0 & 0 & 10 & 65.3 & 2.10 & 1.24 \\
\hline 10 & & - & LWZ & 0.5 & 0 & 0 & 10 & 82.0 & 2.15 & 1.24 \\
\hline 11 & & - & LWZ & 0.7 & 0 & 0 & 10 & 82.3 & 1.88 & 1.04 \\
\hline 12 & & - & LWZ & 0.9 & 0 & 0 & 10 & 83.8 & 2.00 & 1.01 \\
\hline 13 & & - & LWZ & 0.1 & 2 & 0 & 10 & 17.3 & 0.87 & 0.78 \\
\hline 14 & & - & LWZ & 0.3 & 2 & 0 & 10 & 50.0 & 1.36 & 1.21 \\
\hline 15 & & - & LWZ & 0.5 & 2 & 0 & 10 & 70.0 & 1.51 & 0.99 \\
\hline 16 & & - & LWZ & 0.7 & 2 & 0 & 10 & 76.4 & 1.47 & 0.97 \\
\hline 17 & & - & LWZ & 0.9 & 2 & 0 & 10 & 71.2 & 1.83 & 0.94 \\
\hline 18 & & - & LWZ & 0.5 & 2 & -5 & 10 & 30.8 & 1.37 & 1.28 \\
\hline 19 & & - & SDS & 0.05 & 0 & 0 & 10 & 0.0 & - & - \\
\hline \multirow[t]{2}{*}{20} & & - & LWZ & 0.05 & & & & & & \\
\hline & & & $+\mathrm{SDS}$ & 0.1 & 0 & 0 & 10 & 77.0 & 2.56 & 1.24 \\
\hline $21^{b}$ & & - & LWZ & 1.5 & 2 & 0 & 10 & 0.0 & - & - \\
\hline $22^{\mathrm{b}}$ & AAPH & $1.0 \times 10^{-3}$ & LWZ & 0.5 & 0 & 0 & 10 & 84.0 & 2.67 & 1.24 \\
\hline $23^{b}$ & AAPH & $1.0 \times 10^{-3}$ & LWZ & 0.5 & 2 & 0 & 10 & 74.1 & 2.36 & 1.10 \\
\hline
\end{tabular}

a Irradiated with a lamp inside of the bath, in the other cases irradiated with a lamp outside of the bath.

b Irradiated with $365 \mathrm{~nm}$ light through UV-DIB filter, in the other cases irradiated with 300, 313, and $365 \mathrm{~nm}$ light without filter.

13700 and 12800 , respectively (Table $\mathrm{I}(18)$ ). The values of $P_{n}$ (PVA) $/ P_{n}$ (PVAc) at 5,0 and $-5^{\circ} \mathrm{C}$ were 0.404 (Table I(1)), 0.656 (Table $\mathrm{I}(15)$ ), and 0.934 (Table $\mathrm{I}(18)$ ), respectively. The high value at $-5^{\circ} \mathrm{C}$ showed that the chain transfer reaction to polymer hardly took place at $-5^{\circ} \mathrm{C}$. However, $P_{n} \mathrm{~s}$ of PVAc and PVA were similar to those obtained at $0^{\circ} \mathrm{C}$. The result suggested the influence of $\mathrm{NaCl}$ on the emulsion polymerization.

\section{Effect of $\mathrm{NaCl}$}

Initially it was expected that PVAc with larger $P_{n}$ would to obtained because the addition of $\mathrm{NaCl}$ decreases concentration of $\mathrm{VAc}$ in water and polymerization in micell occurs predominantly. However, as shown in
Table I(13-17), $\mathrm{NaCl}$ did not improve yield and $P_{n}$ but retarded them. The marked retardation at low LWZ concentration suggested that $\mathrm{NaCl}$ made micell unstable. So it was found that $\mathrm{NaCl}$ was of no use except for an antifreezing reagent.

\section{Effect of SDS}

As above mentioned, PVAc of large $P_{n}$ was obtained at low LWZ concentration because of decreasing of initiation rate by LWZ. In order to decrease LWZ amount and to keep emulsion stable, SDS, which can not initiate the emulsion polymerization by photo-irradiation (Table I(19)), was added. As shown in Table I( 7 and 20$)$, the yield became twice by the addition of $0.1 \mathrm{ml}$ of SDS, and $P_{n}$ of PVAc 

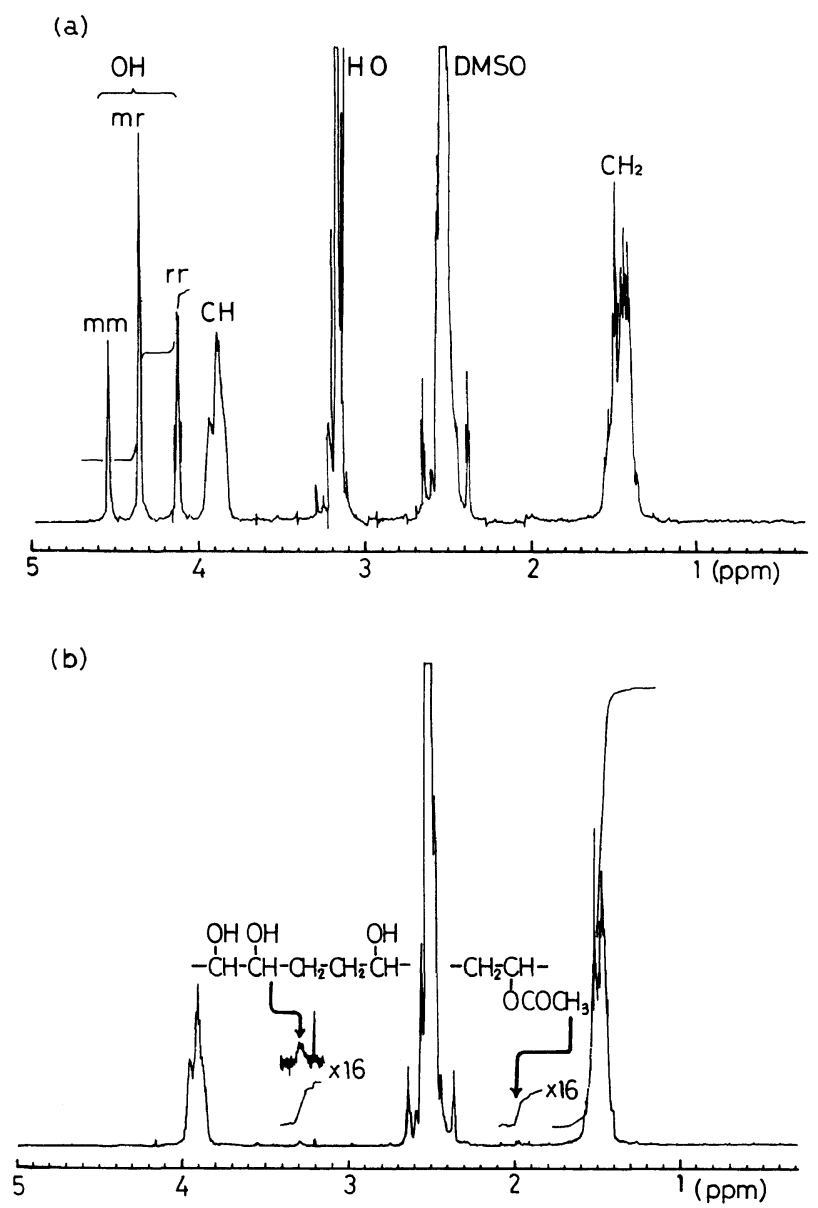

Figure 1. ${ }^{1} \mathrm{H}$ NMR spectra of PVA obtained through photo-emulsion polymerization measured in DMSO- $d_{6}(\mathrm{a})$ and in DMSO- $d_{6}$ in the presence of trifluoroacetic acid.

increased a little but $P_{n}$ of PVA was not changed.

\section{Dependence on Wavelength}

Although PVAcs were obtained in good yield by irradiation with 365,313 , and $300 \mathrm{~nm}$ lights in the absence of an initiator, no polymerization occured with $365 \mathrm{~nm}$ light through a filter (UV-DIB; Toshiba Co.). So $365 \mathrm{~nm}$ light is not effective in the photo-emulsion polymerization of VAc in the absence of initiator. However, when AAPH (water soluble azo-compound) was added, PVAcs of large $P_{n}$ were obtained in good yield by irradiation with $365 \mathrm{~nm}$ light
(Table I(21-23)).

\section{Characteristics of $P V A$}

The characteristics of the typical PVA obtained as above were analyzed as following. The ratios of triad tacticity were estimated as $22.1,49.5$ and $28.4 \%$ for isotactic, heterotactic and syndiotactic triad, respectively, from the three peaks in the $\mathrm{OH}$ proton spectrum shown in Figure 1(a). The ratio of 1,2-glycol structure was estimated to be about $1 \%$ from the ratio of the intensity of methine proton of the structure at $3.3 \mathrm{ppm}$ to that of methylene proton at $1.5 \mathrm{ppm}$ in Figure 1(b) observed in 


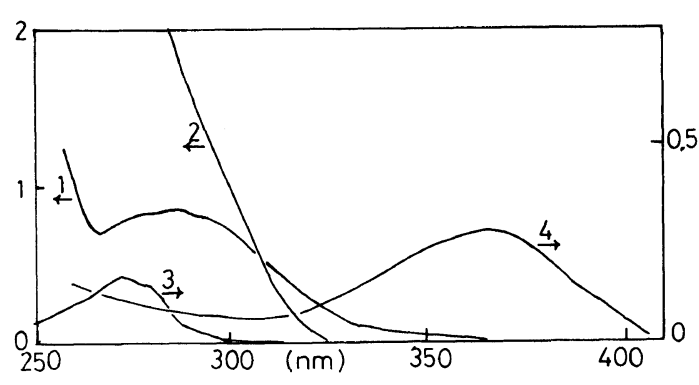

Figure 2. UV-spectra of VAc, 1; Pyrex glass, 2; LWZ, 3; AAPH, 4.

the presence of trifluoroacetic acid. The degree of saponification was estimated to be $99.7 \%$ from the ratio of the intensity of acetyl proton at 2 ppm to that of methylene proton in Figure 1(b). These values of characteristics are similar to those of PVA obtained usually through radical polymerization of VAc.

\section{On the Initiation Mechanism}

As above described polymerization took place by irradiation with 313 and $300 \mathrm{~nm}$ lights in the absence of initiator, when LWZ was used as an emulsifier. But, when SDS was used as an emulsifier, polymerization did not take place. These facts indicated that vinyl acetate monomer did not initiate but LWZ initiated the photo-emulsion polymerization. So we considered the initiation mechanism as following: LWZ has a very low absorbance at 313 and $300 \mathrm{~nm}$ (Figure 2) and hardly decomposes by irradiation. However, once it decomposes, the hydrophilic part is pulled out into the aqueous phase, and another hydrophobic part is pulled into monomer phase in the micelle to initiate polymerization efficiently. Radicals are too few to terminate and $P_{n}$ of PVAc is controlled mainly by chain transfer reaction. As the results, PVAc of large $P_{n}$ is obtained, but the initiation mechanism has not been well clarified. We are investigating the initiation mechanism and will present it elsewhere.

Acknowledgments. The authors are grateful to Dr. T. Okaya and Mr. T. Satoh, Kuraray Co., Ltd. for their help, and also thank to Kao Co. A part of this work was supported by a Grant-in-Aid from the Ministry of Education, Science, and Culture of Japan.

\section{REFERENCES}

1. I. Sakurada, T. Ito, and K. Nakamae, J. Polym. Sci., C15, 75 (1966).

2. I. Sakurada and O. Yoshizaki, Kohbunshi Kagaku, 14, 339 (1957); S. Nozakura, Y. Morishima, H. Iimura, and Y. Irie, J. Polym. Sci., Polym. Chem. Ed., 14, 759 (1976); Y. Morishima, W. Kim, and S. Nozakura, Polym. J., 8, 196 (1976).

3. T. Yamamoto, S. Seki, M. Hirota, and M. Kamachi, Polym. J., 19, 1417 (1987).

4. A. Nakajima, Kobunshi Kagaku, 11, 142 (1954). 